University of Nebraska - Lincoln

DigitalCommons@University of Nebraska - Lincoln

May 1993

\title{
Light diffraction by field-induced non-periodic magnetic domain structures in $\mathrm{FeCl} 2$
}

Christian Binek

University of Nebraska-Lincoln, cbinek@unl.edu

Follow this and additional works at: https://digitalcommons.unl.edu/physicsbinek

Part of the Physics Commons

Binek, Christian, "Light diffraction by field-induced non-periodic magnetic domain structures in FeCl2" (1993). Christian Binek Publications. 50.

https://digitalcommons.unl.edu/physicsbinek/50

This Article is brought to you for free and open access by the Research Papers in Physics and Astronomy at DigitalCommons@University of Nebraska - Lincoln. It has been accepted for inclusion in Christian Binek Publications by an authorized administrator of DigitalCommons@University of Nebraska - Lincoln. 


\title{
Light diffraction by field-induced non-periodic magnetic domain structures in $\mathrm{FeCl}_{2}$
}

\author{
C Binek and W Kleemann \\ Angewandte Physik, Universität Duisburg, W-4100 Duisburg 1, Federal Republic of Germany
}

Received 21 January 1993

\begin{abstract}
The magnetic-field-induced mixed phase of the metamagnet $\mathrm{FeCl}_{2}$ gives rise to reduced transmittivity of circularly polarized light. Within the framework of the diffraction theory of thin random magnetic phase gratings and the assumption of field-dependent refractive indices of the antiferromagnetic domains the field dependence of the transmission is perfectly modelled.
\end{abstract}

\section{Introduction}

Experiments which study the transmission of light are a very precise method for determining the mixed-phase boundaries of metamagnets [1]. As shown by Dillon et al [2] the field dependence of the transmitted light intensity is caused by light diffraction. They also explained the apparent dichroism which was introduced previously [1] by the use of different refraction index contrasts for left and right circularly polarized light. Dillon et al [2] gave only a qualitative explanation for their transmission experiments, stressing the analogy to periodic phase gratings as analyšed for example by Kuhlow [3].

The aim of this paper is to describe quantitatively the transmitted intensity as a function of an external field in the Fraunhofer diffraction limit. In pärticular, we avoid the simplified assumption of a regular phase grating but introduce a general non-periodic spatial distribution of two different refraction indices.

\section{Experimental details}

A parallel beam of circularly polarized laser light at $670 \mathrm{~nm}$ was pulsed at a modulation frequency of $70 \mathrm{kHz}$ and transmitted perpendicularly through a thin (0001) sheet of $\mathrm{FeCl}_{2}$. The crystals were Bridgman grown and prepared in a dry helium atmosphere. The sample was mounted in a cryomagnet, allowing for axial magnetic fields $B$ up to $5 \mathrm{~T}$ and temperatures between 3.6 and $300 \mathrm{~K}$ stabilized to within $2-10 \mathrm{mK}$. The central spot of the transmitted light was selected by the use of an aperture. Light within a cone of $5 \times 10^{-3} \mathrm{rad}$ was detected with a photomultiplier tube. The first harmonic of the light intensity signal was analysed using the lock-in technique. After zero-field cooling the transmitted intensity was recorded at a constant temperature $T<T_{\mathrm{N}}=23.7 \mathrm{~K}$ as a function of the applied field, $0 \leqslant B \leqslant 3 \mathrm{~T}$. Following the notation in [2] we distinguish between the + and polarizations of the circularly polarized light with respect to the direction of magnetization. The two polarization states were produced by the use of a quarter-wave plate. 


\section{Experimental results}

Figure 1 shows the field dependences of the transmitted intensities (open circles) of + (curve $(a)$ ) and - (curve $(b)$ ) polarized light measured for $\mathrm{FeCl}_{2}$ at $T=10 \mathrm{~K}$. The data are normalized to their respective maximum $I_{0}=I(B<1.1 \mathrm{~T})$. Within the mixed-phase region $B_{\mathrm{c} 1}=1.06 \mathrm{~T} \leqslant B \leqslant B_{\mathrm{c} 2}=1.75 \mathrm{~T}$, the intensities drop by $35 \%$ and $60 \%$ for curves $(a)$ and $(b)$, respectively, owing to diffraction losses [2]. Furthermore, the parabola-shaped loss functions $\Delta I=I-I_{0}$ versus $B$ are asymmetric, the steepest slopes occurring at $B_{\mathrm{cl}}$ and $B_{\mathrm{c} 2}$ for + and - polarized light, respectively [2].

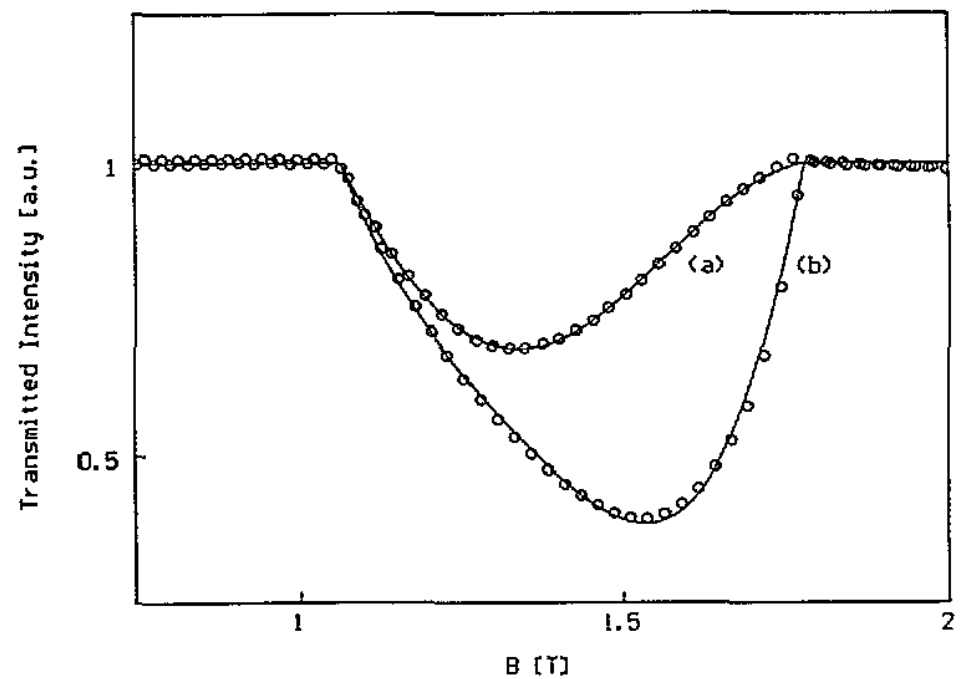

Figure 1. Transmitted intensity versus applied field $(O)$ for + polarized light (curve $(a)$ ) and - polarized light (curve $(b)$ ) measured for $\mathrm{FeCl}_{2}$ at $T=10 \mathrm{~K}$ and least-squares fits (-) by the use of equation (17) (a.u., arbitrary units).

\section{Theory and comparison with experimental results}

The aim of the following analysis is to determine an expression for the diffraction pattern of a two-dimensional distribution of two different refraction indices within an infinitely large plane-parallel thin transparent sample. Hence, we neglect diffraction due to the finite size of a real sample, which is hit perpendicularly by the incident coherent plane light wave. Furthermore we restrict the calculation to the Fraunhofer diffraction limit. According to Moharam et al [4], we can estimate that we are slightly outside the Bragg regime. Because of the smallness of the parameter $\rho=(\lambda / \Lambda)^{2} /\left(n_{0} n_{1}\right)<5$ with a ratio $\lambda / \Lambda$ of the wavelength to the grating periodicity of about 0.07 , the average refraction index $n_{0}>1$ and the index grating amplitude $n_{1} \simeq 10^{-3}$ we are outside the Bragg regime, which requires $\rho>10$ [4]. This means that we do not have to use the theory of thick magnetic phase gratings.

From the convolution theorem [5] we obtain the transmitted intensity $I(u)$ as

$$
I(\boldsymbol{u}) \sim \int_{-\infty}^{\infty} K(\boldsymbol{r}) \exp (-\mathrm{i} \boldsymbol{u} \cdot \boldsymbol{r}) \mathrm{d} x \mathrm{~d} y
$$


with $u=(2 \pi / \lambda)(\sin \xi, \sin \eta), \lambda$ the wavelength of the incident light, $r=(x, y)$ the sample plane coordinate and $\xi, \eta$ the coordinates referring to the Fraunhofer plane. $K(r)$ is the spatial correlation function defined as

$$
K(r)=\lim _{a \rightarrow \infty}\left(\frac{1}{a^{2}} \int_{-a / 2}^{a / 2} E\left(r^{\prime}\right) \cdot E\left(r^{\prime}-r\right) \mathrm{d} x^{\prime} \mathrm{d} y^{\prime}\right)
$$

with the electric field strength $E(r)$ immediately after transmission through the sample. $E(r)$ depends on the special realization of the index distribution which we assume to be random. However, the correlation function implies a spatial average which can be substituted by the probability average.

In order to find an analytic expression for $K(r)$ we examine an arbitrary position $r$ of the sample. At this position the normalized electric field strength $E(r) / E_{0}$ can be either $A$ or $B$ depending on the bimodal index distribution realized, where $|A|^{2}=|B|^{2}=1 . E_{0}$ refers to the homogeneously polarized field amplitude prior to transmission, whereas $\boldsymbol{A}$ and $\boldsymbol{B}$ are the differently polarized fields just after passing regions with weak and strong Faraday rotation due to low (antiferromagnetic) and high (saturated paramagnetic) magnetization, respectively. The normalized correlation function then yields $K(r=0)=1$. Let $p_{1}$ be the probability of finding the field $A$ and $p_{2}$ the probability of finding the field $B$.

For the special purpose of domain diffraction of the mixed phase we assume that the paramagnetic volume fraction increases linearly with increasing external magnetic field $B$. Then we obtain

$$
p_{2}(B)=\left\{\begin{array} { l } 
{ 0 } \\
{ ( B - B _ { \mathrm { c } 1 } ) / ( B _ { \mathrm { c } 2 } - B _ { \mathrm { c } 1 } ) } \\
{ \mathrm { I } }
\end{array} \quad \text { if } \left\{\begin{array}{l}
B \leqslant B_{\mathrm{c} 1} \\
B_{\mathrm{c} 1}<B<B_{\mathrm{c} 2} \\
B \geqslant B_{\mathrm{c} 2}
\end{array}\right.\right.
$$

and $p_{1}=1-p_{2}$. Note that $B_{\mathrm{c} 2}>B_{\mathrm{c} 1}$ whenever demagnetization gives rise to smearing of the phase transition as is the case for a platelet-shaped thin sample.

Now we assume $|r|$ to be much larger than the typical width $z$ of a homogeneous domain in the mixed phase. Then two regions of the sample separated by $|\boldsymbol{r}|$ are completely independent of each other. The correlation function is then fully determined by the joint probabilities:

$$
K(\boldsymbol{r})=p_{1}^{2}|\boldsymbol{A}|^{2}+2 p_{1} p_{2} \boldsymbol{A} \cdot \boldsymbol{B}+p_{2}^{2}|\boldsymbol{B}|^{2}=1-2 p_{1} p_{2}(1-\cos \psi)
$$

with $\boldsymbol{A} \cdot \boldsymbol{B}=\cos \psi$ and the phase contrast $\psi=2 \pi \Delta n d / \lambda$ which contains the refraction index contrast $\Delta n$ and the thickness $d$ of the sample.

In order to describe the correlation function for all $r$ we make the ansatz of an exponential decay by analogy to the results of small-angle $\mathrm{x}$-ray diffraction on heterogeneous alloys [6]. Owing to the random distribution of the magnetic domains, $K(r)$ is isotropic [7], i.e. $K(r)=K(r)$ where $r=|r|$. Then we have

$$
K(r)=1-2 p_{1} p_{2}(1-\cos \psi)[1-\exp (-r / z)] .
$$

Insertion of equation (5) into proportionality (1) yields

$$
I(u) \sim D \int_{-\infty}^{\infty} \exp (-\mathrm{i} u \cdot r) \mathrm{d} x \mathrm{~d} y+\Omega \int_{-\infty}^{\infty} \exp \left(-\frac{r}{z}\right) \exp (-\mathrm{i} u \cdot r) \mathrm{d} x \mathrm{~d} y
$$


where we introduce

$$
D=1-2 p_{1} p_{2}(1-\cos \psi) \quad \Omega=1-D .
$$

From (6) we obtain, by using the polar coordinates $(r, \varphi)$,

$$
I(u) \sim D(2 \pi)^{2} \delta(u)+\Omega \int_{0}^{\infty} \mathrm{d} r r \exp \left(-\frac{r}{z}\right) \int_{0}^{2 \pi} \mathrm{d} \varphi \exp (-\mathrm{i} u r \cos \varphi)
$$

where $u=|u|$. With [8]

$$
\int_{0}^{2 \pi} \mathrm{d} \varphi \exp (-\mathrm{i} u r \cos \varphi)=2 \pi J_{0}(u r)
$$

where $J_{0}$ is the Bessel function of order zero, we finally obtain by integration

$$
l(u) \sim D(2 \pi)^{2} \delta(u)+\Omega 2 \pi z^{2} /\left[1+(u z)^{2}\right]^{3 / 2} .
$$

For direct comparison with our experimental results we require the intensity IPMT $_{\text {Pat }}$ reaches the photomultiplier. This is the transmitted intensity within a small cone about the normal selected by an aperture. We choose the aperture sufficiently large that diffraction can be neglected but small enough to use the approximations $\sin \xi \simeq \xi$ and $\sin \eta \simeq \eta$. Then $I_{\mathrm{PMT}}$ is given by integration over the aperture

$$
I_{\mathrm{PMT}}=\int \mathrm{d} \xi \mathrm{d} \eta I(u(\xi, \eta)) .
$$

Introduction of the polar coordinates $(\beta, \varphi)$ yields

$$
I_{\mathrm{PMT}} \sim D \lambda^{2}+2 \pi \Omega z^{2} \int_{0}^{2 \pi} \mathrm{d} \varphi \int_{0}^{\alpha} \mathrm{d} \beta \frac{\beta}{\left[1+(2 \pi z \beta / \lambda)^{2}\right]^{3 / 2}}
$$

where $\alpha \simeq a / L$ with $a$ the radius of the aperture and $L$ the distance between the sample and the aperture. Solving the integrals in equation (12) yields

$$
l_{\mathrm{PMT}}=\gamma\left\{D+\Omega\left[1-1 / \sqrt{(2 \pi z \alpha / \lambda)^{2}+1}\right]\right\} \quad \gamma=\text { constant. }
$$

Clearly, the $D$-term in (13) corresponds to the central component at $\xi=\eta=0$, whereas the $\Omega$-term describes that part of the diffracted halo, which passes the diaphragm. In the limit $\alpha \rightarrow 0$ the second term vanishes. It can, hence, be expected that the observed drop in the measured intensity within the mixed phase is primarily due to the dependence of the central component on the domain distribution. This is easily seen by rewriting equation (13) and using the relation (7). We obtain

$$
I_{\mathrm{PMT}}=\gamma\left[1-2 p_{1} p_{2}(1-\cos \psi) / \sqrt{1+(2 \pi z \alpha / \lambda)^{2}}\right] .
$$

Assuming that $z \simeq 5 \mu \mathrm{m}[2,10]$ and choosing $\lambda=0.7 \mu \mathrm{m}$ and $\alpha=1 / 400$ we have $(2 \pi z \alpha / \lambda)^{2} \sim 10^{-2} \ll 1 ;$ hence,

$$
I_{\mathrm{PMT}} \simeq \gamma\left[1-2 p_{1} p_{2}(1-\cos \psi)\right] \text {. }
$$

By inspection of (3) we see that $p_{1} p_{2}$ is a quadratic function of the external field $B$. Inserting the maximum value $p_{1} p_{2}=\frac{1}{4}$ into equation (15) and approximating $\cos \psi \simeq 1-\frac{1}{2} \psi^{2}$ we obtain a quadratic dependence on $\Delta n$ of $\Delta I$ versus $B$. This is expected from general diffraction efficiency calculations [9] and has already been used in [2]. This approximation is clearly not applicable in general because $\psi \ll \frac{1}{2} \pi$ is a restriction for the validity of the above expansion but not of equation (15). 


\subsection{Line-shape asymmetry}

Peculiarly, however, the intensity functions $\Delta I$ versus $B$ reveal polarization-dependent amplitudes and asymmetries as remarked previously [2]. In agreement with [2] we ascertain that the amplitudes are determined by different index contrasts $\Delta n^{+/-}=n_{\mathrm{p}}^{+/-}-n_{\mathrm{a}}^{+/-}$, where $p$ and a refer to the different magnetic phases and $+/-$ to the different circular polarizations.

In contrast with [2], however, we do not believe that the asymmetry can be explained by domain shape effects. If the line-shape asymmetry were merely due to the geometrical properties of the domains, the behaviour should not change in quality by inversion of the polarization from + to - . Rather we believe that the line-shape asymmetry is also a material property. Hence, we introduce a field-dependent refractive index contrast which we allow to differ for the two circular polarization states. Since we do not know the analytical description of this field dependence we introduce a Taylor series up to second order in powers of the field:

$$
\Delta n^{+/-}=\Delta n_{0}^{+1-}+N_{1}^{+/-}(B-b)+N_{2}^{+/-}(B-b)^{2}
$$

with $b=\frac{1}{2}\left(B_{\mathrm{c} 1}+B_{\mathrm{c} 2}\right)$ the field value in the middle of the mixed phase and the expansion coefficients $\Delta n_{0}^{+1-}, N_{1}^{+1-}$ and $N_{2}^{+1-}$.

Normalizing equation (15) to the maximum value $\gamma$ and inserting (16) we obtain the intensity function

$\bar{I}_{\text {PMT }} \simeq 1-2 p_{1} p_{2}\left(1-\cos \left\{(2 \pi d / \lambda)\left[\Delta n_{0}^{+/-}+N_{1}^{+/-}(B-b)+N_{2}^{+/-}(B-b)^{2}\right]\right\}\right)$.

The physics behind expansion (16) is the following. From Faraday rotation measurements it is known that at non-zero temperatures there is a small non-linear increase in the Faraday rotation with increasing field already below the metamagnetic transition. This is due to the growth of the antiferromagnetic refraction index contrast caused by the increase in ferromagnetic fluctuations with increasing field. This precursor effect is similar to that observed in ferroelectrics [11] and is assumed to increase within the mixed phase.

The experimental data within $B_{\mathrm{cl}} \leqslant B \leqslant B_{\mathrm{c} 2}$ in figure 1 have been best fitted to equation (17) by using different sets of fitting parameters for each polarization as listed in table 1 . It is seen that nearly perfect coincidence of experimental and theoretical data is achieved. The negative signs of the parameters $\psi_{1}^{+/-}=(2 \pi d / \lambda) N_{1}^{+/-}$and $\psi_{2}^{+/-}=(2 \pi d / \lambda) N_{2}^{+/-}$ show that the refraction index contrasts decrease with increasing magnetic field for both polarization states. This is what we expect because of the conjectured increase in $\Delta n_{\mathrm{a}}$ with increasing $B$ within the precursor regime.

Table 1. Parameters obtained from least-squares fits of the data in figure 1 to equation (17) for + and - polarized light.

\begin{tabular}{llll}
\hline Polarization state & $\left.\psi_{0}=(2 \pi d / \lambda) \Delta n_{0}\right)$ & $\psi_{1}=(2 \pi d / \lambda) N_{1}$ & $\psi_{2}=(2 \pi d / \lambda) N_{2}$ \\
\hline+ & 1.140 & -1.332 & -1.954 \\
- & 4.604 & -1.823 & -4.476 \\
\hline
\end{tabular}

We also measured the Faraday rotation on the same sample at $T=10 \mathrm{~K}$ and obtain the saturation value $\psi_{\mathrm{s}}$ of the Faraday rotation as $150^{\circ}$. This value is related to the circular birefringence of the paramagnetic phase: $\Delta n_{\mathrm{p}}=n_{\mathrm{p}}^{-}-n_{\mathrm{p}}^{+}$. This is approximately given by $\psi_{\mathrm{d}}=\frac{1}{2}\left(\psi^{+}+\psi^{-}\right)$determined at $B_{\mathrm{cl}}$, where we assume that $n_{\mathrm{a}}^{+} \simeq n_{\mathrm{a}}^{-}$owing to minimal precursor contributions. Inserting $B=B_{\mathrm{cl}}$ into equation (16), one obtains $\psi_{\mathrm{d}}=174^{\circ}$, which is close to the observed value within acceptable error margins. 


\section{Conclusion}

Within the scalar diffraction theory of random circular index gratings we obtain a quadratic field dependence of the transmitted intensity. The observed line-shape asymmetry is a nonnegligible effect which is very probably caused by a field dependence of the refraction index contrast. It is therefore not appropriate to use the ratio of the intensity drop $\Delta I$ to determine the relation of the refraction index contrasts for + and - polarized light.

Contrary to this it is in principle possible to obtain separated information about the phase contrasts $\psi^{+/-}$by fitting equation (17) to the experimental data. Nevertheless determination of the corresponding refraction index contrasts remains difficult owing to uncertainties in the sample thickness and ambiguities caused by the periodicity of the cosine term in equation (17).

\section{Acknowledgment}

This work was supported by Deutsche Forschungsgemeinschaft through Sonderforschungsbereich 166.

\section{References}

[1] Griffin J A, Schnatterly S E, Farge Y, Regis M and Fontana M P 1974 Phys. Rev, B 101960

[2] Dillon J F Jr, Yi Chen, Guggenheim H J and Alben R 1977 Phys. Rev. B 151422

[3] Kuhlow B 1979 Optik $\mathbf{5 3} 115$

[4] Moharam M G, Gaylord T K and Magursson R 1980 Opt. Commun. 3214

[5] Cowley J M 1981 Diffraction Physics (Amsterdam; North-Holland) p 30

[6] Glatter O and Kratky O 1982 Small Angle X-ray Scattering (London: Academic) p 296

[7] Guinier A 1963 X-ray Diffraction (San Francisco, CA: W H Freeman) p 45

[8] Abramowitz M and Stegun I A 1972 Handbook of Mathematical Functions (New York: Dover) p 498

[9] Magnusson R and Gaylord T K 1978 J. Opt. Soc. Am. 68806

[10] Dillon J F Jr, Yi Chen and Guggenheim H J 1975 Solid State Commun. 16 371

[11] Kleemann W, Schäfer F J and Rytz D 1986 Phys. Rev. B 347873 